contents have been discussed in a previous abstract. The survey was completed upon request and in the presence of the reference clinician throughout the last quarter of 2017. The survey assessed three areas: therapeutic and hygienic-dietetic adherence, the perception of personalized follow-up and overall practical utility. In parallel, the number of annual attacks of patients using ReumaApp-Gota and the relative annual reduction of the uric acid level in peripheral blood were determined. In the case of patients using ReumaApp-AR, the demand for unscheduled visits due to flares of RA and the relative reduction of DAS28 were evaluated. In both cases, their results were compared with cohorts of patients who had not used any of the applications (35 of the drop group and 29 of the AR group).

Results: Users of ReumAPP-Gota: Improvement in adherence 25/65, personalized follow-up is perceived 56/65, has utility in day-to-day 52/65. Users of ReumAPP-AR: Improve adherence $31 / 35$, personalized follow-up is perceived $30 / 35$, has utility in day to day $29 / 35$. The number of annual gout attacks in patients users of the APP was 1.32 OF 0.45 and that of non-users was 1.27 OF $0.52(P=0.848)$, the relative annual reduction of patients with gout users of the APP was $36.6 \mathrm{OF}$ $6.6 \%$ and that of non-users 33.7 of $11.2 \%(P=0.106)$. The number of urgent visits in a year of patients with RA users of the APP was 0.55 of 0.12 and that of the nonusers group 0.83 of $0.29(\mathrm{P}<0.001)$. The relative reduction of the DAS28 of patients using the APP was 34.9 of $5.9 \%$ and of the non-users was 31 of $7.7 \%$ $(\mathrm{P}<0.025)$.

Conclusions: Although the use of applications for smartphones seems to generate a greater sense of medical vigilance on the part of patients and is globally considered useful, its impact on the therapeutic adherence in patients with gout is low and is reflected in the objective results of reduction of plasma uric acid figures and the number of annual attacks. In the case of patients with RA the use of the application significantly reduces the need for urgent visits, most likely because it allows the clinician to contact the patient priorly when certain parameters entered exceed certain margins. But on the other hand the relative reduction of DAS28 may be due to the fact that patients not users of the application are usually older than those who accept its use.

Disclosure of Interest: None declared

DOI: 10.1136/annrheumdis-2018-eular.7314

\section{FRI0614 PHYSICIAN-PATIENT INTERACTIONS IN AFRICAN AMERICAN PATIENTS WITH SYSTEMIC LUPUS ERYTHEMATOSUS}

C. Drenkard 1 , G. Bao ${ }^{1}$, T. T. Lewis ${ }^{2}$, B. F. Pobiner ${ }^{3}$, J. Priest ${ }^{3}$, S. S. Lim¹. ${ }^{1}$ Rheumatology, ${ }^{2}$ Epidemiology, Emory University, Atlanta, ${ }^{3}$ GlaxoSmithKline, North Carolina, United States

Background: African American (AA) patients with systemic lupus erythematosus (SLE) are at high risk for poor outcomes. Patient characteristics and disease severity influence physician-patient interactions, which in turn can impact outcomes.

Objectives: Examine the relationships between physician-patient interactions and demographics, disease activity, and depression in AA SLE patients.

Methods: The Georgians Organized Against Lupus (GOAL) is a populationbased cohort of patients with a documented diagnosis of SLE. We conducted a cross-sectional analysis of patient-reported data among 698 AA participants (out of 863 GOAL participants). We assessed patients reports of physician-patient interactions (communication, patient-centered decision making, and physician interpersonal style) through the Interpersonal Processes of Care survey (IPC-29), disease activity through the Systemic Lupus Activity Questionnaire, and depression through the Patient Health Questionnaire-9. We used non-parametric tests to assess IPC-29 by demographics, linear trend test to assess demographicadjusted scores of IPC-29 by disease activity and depression, and multivariate logistic regression to assess the association of disease activity and depression with suboptimal IPC scores.

Results: Lowest IPC-29 scores were for patient-centered decision making, specifically for the "asked patient" subdomain (how often doctors assessed patients' problems to follow recommendations and treatment) in women compared with men (mean score $3.1 \pm 1.4$ and $3.6 \pm 1.4$, respectively; $p=0.02$ ). Scores for the "assumed socioeconomic level" subdomain (how often doctors make assumptions about a patient's socioeconomic level) in the interpersonal style domain were higher (worse) in patients aged 18-34 (mean score 1.6 \pm 0.9 ), compared to those aged $35-55$ (mean score $1.5 \pm 0.9 ; p=0.03$ ). Patients with higher educational attainment $(>$ college) reported poorer mean scores for most communication and interpersonal style scales than those with $\leq$ high-school. We found significant linear trends of poorer scores for all communication scales across more severe disease activity and depression, and poorer scores for all interpersonal style scales across more severe disease activity. Neither disease activity nor depression was associated with worse patient-centered decision-making. In multivariate models depression was associated with suboptimal quality of communication (OR 1.2; 95\% Cl 1.04-1.4) and interpersonal style (OR 1.1; 95\% Cl 1.0-1.3); and dis ease activity increased the odds of suboptimal communication (OR $1.1 ; 95 \% \mathrm{Cl}$ 1.0-1.3).

Conclusions: In AA patients with SLE, perceptions of physicians' communication, shared decision-making, and physicians' interpersonal style vary by sex and education. While disease activity and depression were associated with worse reports of physician-patient communication and interpersonal style, neither disease activity nor depression influenced shared-decision making. Our data sug gest that in addition to standard of care treatment, AA SLE patients with active disease and depression might need provider-based interventions focused on communication and interpersonal style.

Acknowledgements: Supported by Human Genome Science Inc. and GlaxoS mithKline (GHO-11-3366), and the Centers for Disease Control and Prevention (CDC-U01DP005119).

Disclosure of Interest: C. Drenkard: None declared, G. Bao: None declared, T. Lewis: None declared, B. Pobiner Shareholder of: GlaxoSmithKline, Employee of: GlaxoSmithKline, J. Priest Shareholder of: GlaxoSmithKline, Employee of: GlaxoSmithKline, S. S. Lim: None declared

DOI: 10.1136/annrheumdis-2018-eular.1210

\section{FRI0615 \\ NORMALISED TOXICITY TO PREGABALIN DID NOT INCREASE WITH CHANGES IN APPROVAL MECHANISM AND USE IN AUSTRALIA}

C. McMaster ${ }^{1}$, D. F. L. Liew ${ }^{1,2,3}$, E. Joules ${ }^{4}$, J. Robinson ${ }^{5}$, S. Greene ${ }^{5}$, R. R. C. Buchanan ${ }^{2,3}$, A. G. Frauman ${ }^{1,3} .{ }^{1}$ Clinical Pharmacology and Therapeutics, ${ }^{2}$ Rheumatology, Austin Health, Heidelberg, ${ }^{3}$ Medicine, University of Melbourne, Parkville, ${ }^{4}$ Pharmacy, ${ }^{5}$ Victorian Poisons Information Centre, Austin Health, Heidelberg, Australia

Background: Pregabalin is a potent antagonist of neuronal voltage-gated calcium channels used to treat neuropathic pain. Although first registered with the Therapeutic Goods Administration (TGA) in April 2005, rapid uptake of pregabalin in the Australian market only came after its listing on the Pharmaceutical Benefits Scheme (PBS) with a streamlined code on 1st March 2013 for the indication of neuropathic pain. Recently, there has been concern raised about the growing off label use of pregabalin for chronic pain syndromes and the subsequent potential for undue adverse drug reactions (ADRs).

Objectives: The aim of this study was to measure how broadening access to pregabalin in Australia affected the rate of pregabalin-associated ADRs, and whether such an effect was disproportionate to the change in pregabalin prescription rates.

Methods: We extracted ADRs reported in the TGA Database of Adverse Event Notifications (DAEN) between 1st January 2009 and 18th October 2017, in which pregabalin was thought to have been causative. We also extracted calls to the Victorian Poisons Information Centre (VPIC) between 1st January 2009 and 31st December 2017 in which pregabalin was a reported exposure. For both data bases, ADR rates were annualised, with the missing DAEN ADR reports between 19th October 2017 and 31st December 2017 imputed using a linear model based on year-to-date ADR rates. The annual ADR rates were normalised by dividing by the estimated number of pregabalin prescriptions filled (in millions), to obtain a normalised Toxicity Index (number of ADRs per million scripts). Because the data was annualised, the 1st January 2013 was used as the approximate starting date of PBS streamlined listing.

Results: The estimated number of pregabalin prescriptions filled in Australia increased over the study period from 155,336 in 2009 to 3,739,421 in 2017. A total of 886 ADRs were reported to VPIC over the study period, and 1056 reported to DAEN (1076 after extrapolation). The mean Toxicity Index (TI) for the VPIC database was 539 ADRs/million scripts before PBS streamlined listing, and 298 ADRs/million scripts after; there was no evidence that the TI had increased ( $p=0.9$, one-tail t-test). Similarly, the TI for the DAEN database was $441 \mathrm{ADRs} / \mathrm{mil}$ lion scripts prior to PBS streamlined listing, versus 85 ADRs/million after; there was no evidence that $\mathrm{Tl}$ increased ( $\mathrm{p}=0.98$, one-tail t-test). 